\title{
Diffusion analysis with high and low concentration regions by finite difference method, adaptive network-based fuzzy inference system, and bilayered neural network method
}

Qike Shao ${ }^{a}$, Sina Faizollahzadeh Ardabilib, Majdi Mafarja ${ }^{\mathrm{c}}$, Hamza

Turabieh $^{\mathrm{d}}$, Qian Zhang ${ }^{\mathrm{e} *}$, Shahab S. Band ${ }^{\mathrm{b} *}$, Kwok-Wing Chau ${ }^{\mathrm{f}}$, Amir Mosavi $^{\mathrm{g}, \mathrm{h}}$

${ }^{a}$ Department of Computer Science and Artificial Intelligence, Wenzhou University, Wenzhou 325035, China; ${ }_{-}^{b}$ Future Technology Research Center, National Yunlin University of Science and Technology, 123 University Road, Section 3, Douliou, Yunlin 64002, Taiwan; ' Department of Computer Science, Birzeit University, POBox 14, West Bank, Palestine; ${ }_{-}^{d}$ Department of Information Technology, College of Computers and Information Technology, Taif University, P.O. Box11099, Taif 21944, Saudi Arabia; ${ }_{-}$ Wenzhou University of Technology, Wenzhou 325035, China; ${ }^{f}$ Department of Civil and Environmental Engineering, The Hong Kong Polytechnic University, Hong Kong, China; ${ }_{-}^{g}$ Faculty of Civil Engineering, Technische Universität Dresden, Dresden, Germany; ${ }^{h}$ John von Neumann Faculty of Informatics, Obuda University, Budapest, Hungary;

\begin{abstract}
The diffusion of molecules in aqueous solutions in the domain of membrane technology is very critical in the efficiency of chemical engineering and purification processes. In this study, the diffusion in high and low concentration regions is simulated with finite difference method (FDM), and then the results of numerical computations are coupled with adaptive network-based fuzzy inference system (ANFIS) and bilayered neural network method (BNNM). Machine learning
\end{abstract}


approach can individually predict diffusion phenomena across the domain based on understanding of the machine instead of the discretization of an ordinary differential equation (ODE). The findings of the machine learning method are in good agreement with those of FDM at different times of the simulation. In addition to numerical computation, the error of the system is computed for different iterations. The results show that by increasing the number of iterations and training datasets, all errors reduce significantly for both training and testing. BNN method is also used to train the prediction process of diffusion for a more accurate comparison. This technique is similar to ANFIS method in terms of prediction capability. According to the findings, ANFIS approach predicts diffusion slightly better than BNN method. In this regard, ANFIS technique produces $\mathrm{R}>0.99$ while BNN method produces $\mathrm{R}$ around 0.98 . Both machine learning methods are accurate enough to predict diffusion throughout the domain for different time steps. The computational time for both algorithms is less than that of FDM method to predict low and high concentrations in the domain. Besides, based on the results, artificial intelligence (AI) can find the relationship between inputs and outputs and determine which input has the main influence on the output in this study to optimize the process. As such, future studies can be focused on AI and other methods for faster prediction and optimization processes.

Keywords: ANFIS; Bilayered neural network; diffusion phenomena; diffusion of molecules; machine learning

\section{Introduction}

Microorganisms, plants, as well as animals are some examples that the role of diffusion of large and small molecules in aqueous solutions is inevitable. Aside from these, the 
process of diffusion has its own role in food processing as well as drying of liquid mixtures and solutions. Examples include aroma as well as flavor components in tea and coffee during the process of evaporation. Moreover, in the process of fermentation, diffusion occurs. In this process, sugar, oxygen as well as nutrients diffuse to products, microorganisms, and waste. In this regard, kidneys are responsible for taking away different products, including creatinine, urea, and other excess fluid from the blood. Besides, kidney dialysis helps patients whose kidneys work improperly to remove waste products from their blood. During the process of hemodialysis, a dialyzer is applied, and in this process, blood is pumped; consequently, waste that exists in blood diffuses by a sort of membrane action so that only particular molecules can pass to the aqueous solution cleaning fluid.

The membrane technology is also seen in the process of hemodialysis. Generally, getting into a cell or getting out of a cell, substances which are dissolved or gaseous have to pass through the cell membrane. When particles start spreading, diffusion happens. Particles actually move from a region in which the concentration is high to a low concentration region. In the same vein, membrane technologies are applied for separating the chemical constituents, and therefore, separation processes are designed according to the membrane technology. This technology leads to a novel method not similar to conventional separation methods that have been used in different industries, for instance, the purification industry. The purification method is used to separate various elements, and is applied in small-scale technologies rather than large-scale ones that are more applied with the conventional methods. Comparing the conventional purification methods by the separation process that is used in the membrane technology, the latter has some benefits. To name some, it includes low cost regarding the separation, as well as a modular design. It also does not need high energy demand. This technology is frequently studied in 
different investigations to highlight the importance of purification and separation process (Asadollahzadeh, Raoufi, Rezakazemi, \& Shirazian, 2018; Dai, Noble, Gin, Zhang, \& Deng, 2016; Marjani, Nakhjiri, Adimi, Jirandehi, \& Shirazian, 2020; Padaki, Murali, Abdullah, Misdan, Moslehyani, et al., 2015; Purkait, \& Singh, 2018; S. M. R. Razavi, Shirazian, \& Nazemian, 2016; Rezakazemi, Dashti, Asghari, \& Shirazian, 2017; Rezakazemi, Shirazian, \& Ashrafizadeh, 2012).

Generally, understanding the behavior of fluids in membrane technologies and specifically in membrane modules is not easy, but applying numerical methods is possible for the understanding by taking advantage of computational fluid dynamics (CFD). Nevertheless, the problem with the process of optimizing the membrane technology using CFD is that it takes much time, and the costs regarding the computational procedure are high; therefore, researchers can take advantage of artificial Intelligence (AI) together with CFD for the purpose of modeling the chemical processes. Membrane technology and phase separation are not exceptions to this (Babanezhad, Masoumian, Nakhjiri, Marjani, \& Shirazian, 2020; Rezakazemi, Dashti, Asghari, \& Shirazian, 2017; Rezakazemi, Mosavi, \& Shirazian, 2019).

Nevertheless, regarding optimizing membrane technology via CFD, it is worth mentioning that the process is time-consuming, and the computational costs are not low. By using AI as well as CFD, the chemical processes can be modeled. Examples of such processes are phase separation as well as membrane technology. Using AI besides CFD makes the learning possible by taking advantage of neural networks and point-by-point learning of CFD mesh elements, and the prediction process is possible through the fuzzy logic system (Babanezhad, Masoumian, Nakhjiri, Marjani, \& Shirazian, 2020; Rezakazemi, Dashti, Asghari, \& Shirazian, 2017; Rezakazemi, Mosavi, \& Shirazian, 
2019).

The current research study applies adaptive neuro-fuzzy inference system (ANFIS) with various ANFIS parameters to learn the diffusion process. ANFIS can be considered a type of artificial neural network, and in this method, the principles of fuzzy logic and neural networks are applied. It has been used as an efficient and optimal method. Because of the capacity of fuzzy logic in deciding output parameters and identifying connections between input and output parameters, this technique is popular among AI numerical prediction methods. Furthermore, ANFIS architecture is often utilized in a wider variety of physical and real-world applications (Babanezhad, Masoumian, Nakhjiri, Marjani, \& Shirazian, 2020; Rezakazemi, Dashti, Asghari, \& Shirazian, 2017; Rezakazemi, Mosavi, \& Shirazian, 2019).

In addition to ANFIS method, bilayered neural network (BNN) method is also used to predict the diffusion process across the domain for different time steps. BNN method includes binary weights as well as activations, and by taking advantage of this method, the computational time can be reduced.

It is also worth mentioning that AI methods can be beneficial in order to enhance CFD modeling uses. This study indicate that ANFIS and BNN models can be applied with CFD for the purpose of predicting fluid flow characteristics in different conditions and circumstances. Moreover, in this study, high as well as low concentration regions are simulated by using FDM method. Afterward, by taking advantage of the machine learning methods, the distribution is stored in the domain. The computed concentration at FDM components is present in ANFIS and BNN methods in two stages, including testing as well as training stages. Afterward, for a better understanding of the process, the machine learning method using FDM data set is applied. Moreover, function analysis is used for 
determining the relationship regarding each of the input parameters on the concentration pattern of the study.

Different numbers of MFs for each input are also applied to test the prediction ability of the model. The focus of the study is considered novel due to the emphasis of the influence of inputs on output in the framework of ANFIS method. This study examines the most significant influence that the inputs have on the output; therefore, the effective parameter is the focus of the study, and it can be found according to the influence of membership functions (MFs) on the output. Thus, the degree of MFs is studied as a function of each input parameter. Consequently, a simulation methodology that can be considered as having high-performance is provided in this study that was not present in other traditional models regarding chemical engineering. In addition, ANFIS is examined and compared with BNN method to assess the level of prediction capability for different time steps and computation nodes.

Due to the nature of numerical methods, computations using these methods are timeconsuming; AI is applied and used besides numerical methods for predicting the process. Besides, this study also uses ANFIS and BNN algorithms to predict the process by using AI. It is also worth mentioning that supervised machine learning methods are data-driven. Therefore, the methods are trained based on the inputs and AI can predict other inputs which are in that particular range.

\section{Methodology}

In the present study, the adaptive neuro-fuzzy inference system (ANFIS) is used to predict the diffusion process, and then different ANFIS parameters are used to train the process. 
BNN technique, in addition to ANFIS method, is used to forecast the diffusion process throughout the domain for various time steps.

As mentioned earlier, ANFIS is applied in the study. It stands for an adaptive neurofuzzy inference system. It has the ability to accurately predict the performance of particular systems which have complex as well as nonlinear characteristics (Babanezhad, Masoumian, Nakhjiri, Marjani, \& Shirazian, 2020; Najafi, Faizollahzadeh Ardabili, Shamshirband, Chau, \& Rabczuk, 2018; Pishnamazi, Babanezhad, Nakhjiri, Rezakazemi, Marjani, et al., 2020; R. Razavi, Sabaghmoghadam, Bemani, Baghban, Chau, et al., 2019; Rezakazemi, Dashti, Asghari, \& Shirazian, 2017; Sefeedpari, Rafiee, Akram, Chau, \& Komleh, 2015; Yan, Safdari, \& Kim, 2020). ANFIS can be considered a sort of artificial neural network, and its origin is from Sugeno and Takagi. Based on the mentioned works, there are three kinds of fuzzy reasoning and if-then rules are run in ANFIS structure. The formula presented below shows the function of the $\mathrm{i}^{\text {th }}$ rule:

$$
w_{i}=\mu_{A i}(\text { XDomain }) \mu_{B i}(\text { YTime })
$$

In equation (1), $w_{i}$ refers to the out-coming signal from the node of the second layer of the model. Besides, $\mu_{A i}$ and $\mu_{B i}$ represent the incoming signals from MFs run on inputs to the node of the same layer regarding the mentioned model.

In the next layer, that is the third one, the relative value of each rule refers to the firing strength that is studied. In other words, it is equal to the weight of each layer over the whole quantity of firing strengths regarding all of the rules. The node function is as shown in the following equation:

$$
\overline{w_{l}} f_{i}=\bar{w}_{l}\left(o_{i} X+p_{i} Z+q_{i} V+r_{i} P+S_{i}\right)
$$


In equation (2), $\mathrm{p}_{\mathrm{i}}, \mathrm{q}_{\mathrm{i}}, \mathrm{r}_{\mathrm{i}}$, and $\mathrm{s}_{\mathrm{i}}$ are the if-then rules parameters of the study.

For the next machine learning method, BNN method is used to train the structure of datasets, including computing nodes and time steps. This method is compared with ANFIS method for the evaluation of the prediction ability of ANFIS method. Three layers are considered in the method, and for each of them, the size of the layer is five.

\section{Results}

In the current research study, by taking advantage of the finite difference method, diffusion is studied. Furthermore, the obtained results are categorized as a function of time and computing nodes. The categorized data are trained in ANFIS and BNN algorithms, and they are predicted via AI capability. In general, the AI framework is applied as a substitution method for the finite difference method. In the beginning, the combination of inputs and output in the method is considered. In this regard, two inputs and two MFs for each of the inputs are considered in the model. The structure has four rules, and this structure can model diffusion.

Besides, 65 percent of the data are considered in the training phase, and then all of the datasets, including the 65 percent of the data used in the training phase and the remaining data sets, are used in the testing phase. Each step is evaluated separately, and after the evaluation, the model is prepared for the prediction process. Before the prediction, the sensitivity analysis for the tuning parameters of the ANFIS model is performed on the number of MFs for each of the inputs, the number of iterations for solving the equation, and the number of inputs. After optimizing all of the parameters, the model is prepared for the final prediction.

As shown in Figure 1, the distribution of functions of input number two is studied. As shown in the figure, the functions of input number two have a suitable reaction. All 
functions can be studied in this model. The functions are also considered for input 1 . This model is an adaptive system that learns to operate more efficiently by using connected nodes or neurons in a layered structure that resembles the human brain. Because of its capacity to learn from data, it has been shown that a neural network can be trained to recognize patterns, classify data, and predict future events (here, different concentrations as a function of time and locations). The improvement of the functions is evident, and the function considered in this study is gaussmf. For the training phase of the method, ten epochs are used, and a suitable level of training is achieved. After having studied the functions regarding the inputs, ANFIS predicted data are studied and compared with the finite difference method results.
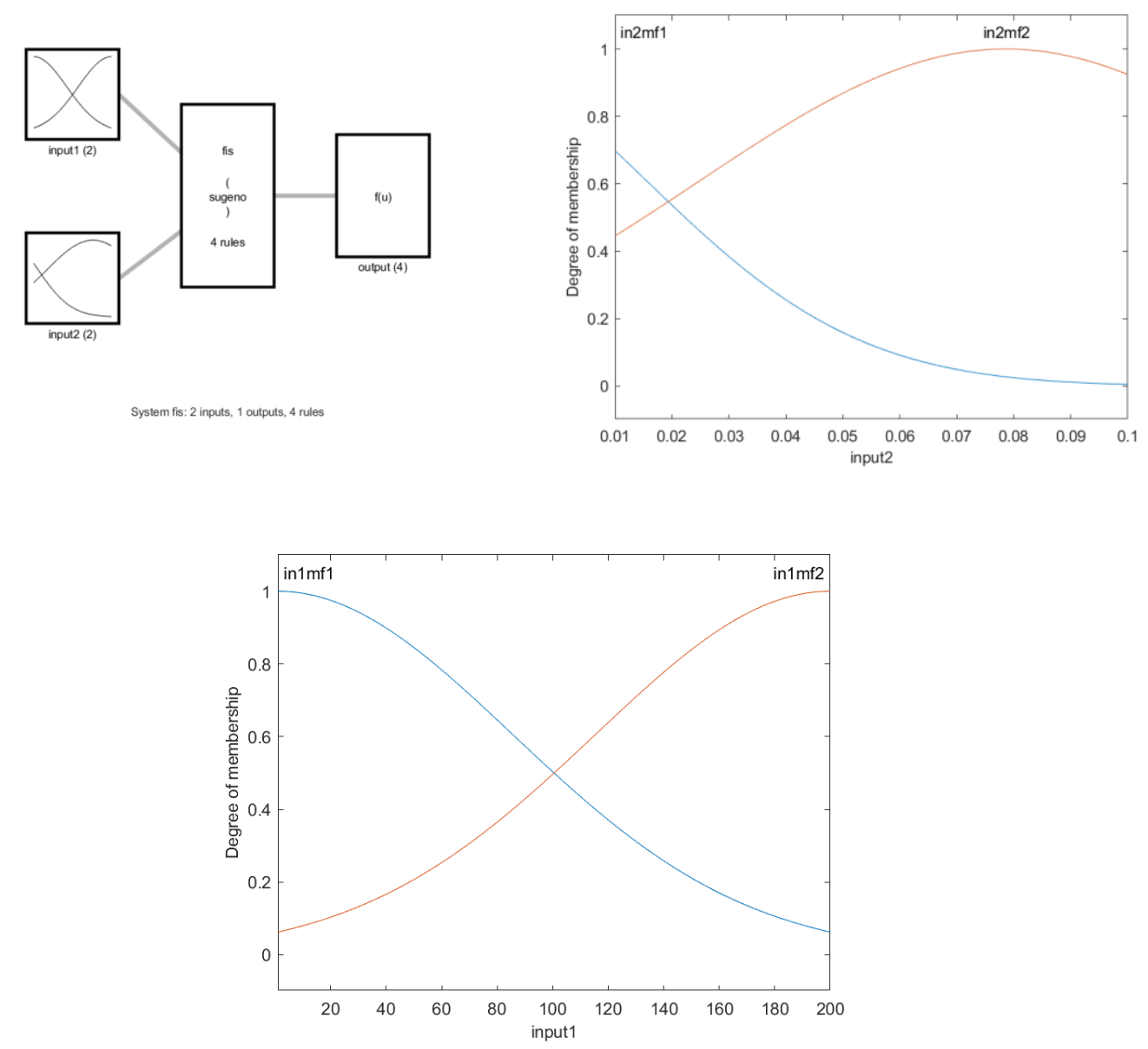
Figure 1. membership function gaussmf of input number two

As shown in Figure 2, ANIFS method has a high capability regarding the prediction and can forecast the behavior simulated by the finite difference method for the diffusion process. Figure 2 also shows the kind of diffusion with respect to time. By considering these results, ANFIS method can capture the diffusion behavior with two MFs, yet more MFs are needed surrounding the membership functions for a better prediction. Alternatively, surrounding boundary conditions need to be used by filtration methods so that ANFIS can better predict them. In this case, ANFIS method can only train datasets far away from the critical boundary condition that impacts the numerical convergence and prediction capability.
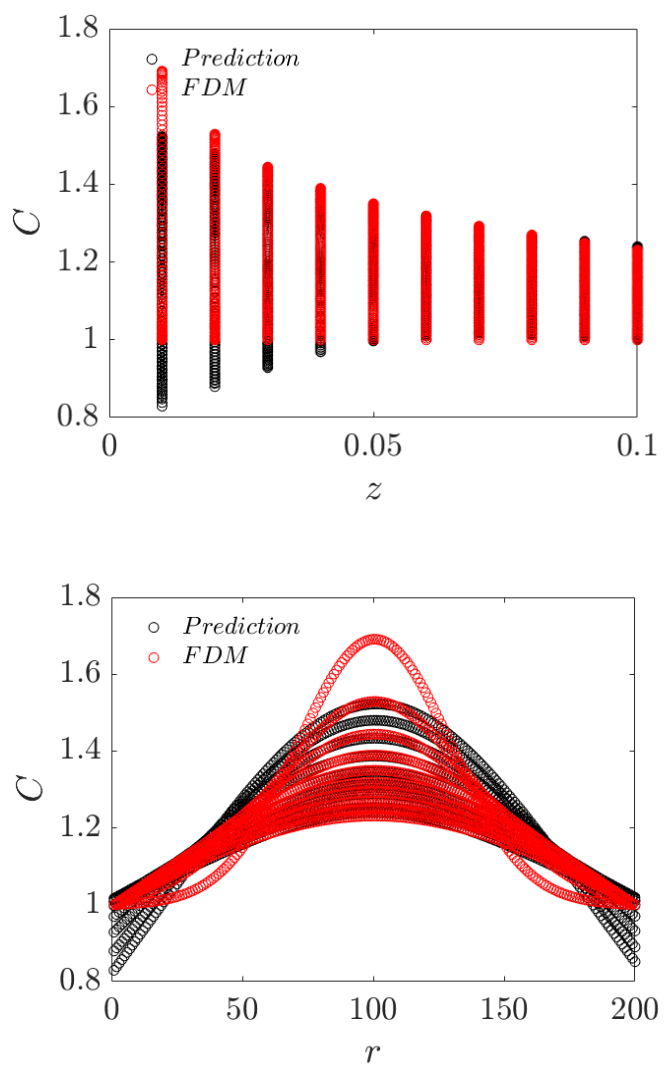
Figure 2. FEM and ANFIS results for membership function gaussmf of input number 2

Figure 3 shows the surface area for diffusion. As shown in this figure, ANFIS can predict the whole diffusion domain. After the prediction by ANFIS, R-value as well as R square are studied.

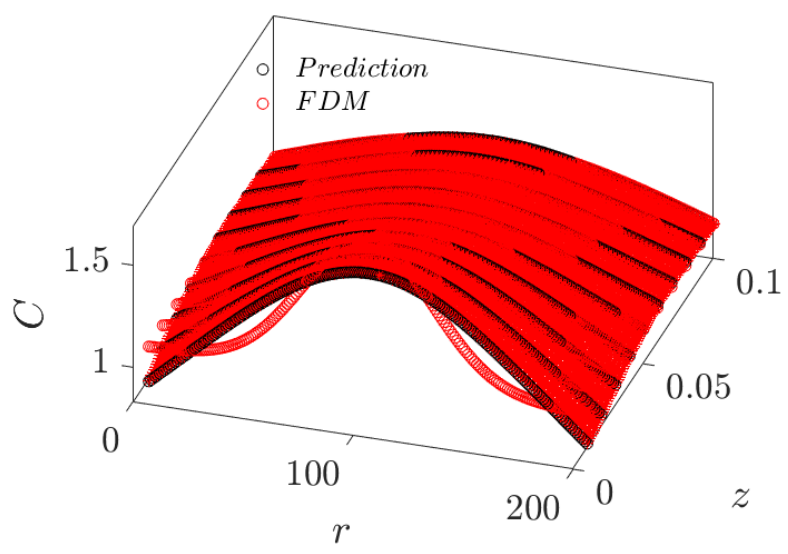

Figure 3. surface plot for membership function gaussmf of input number 2

As shown in Figure 4, R-values in testing and training are equal to 0.96365 and 0.96334 , respectively. As shown in the figure, the testing and training data have similar overall pattern to one another. Furthermore, ANFIS can predict both testing and training stages.
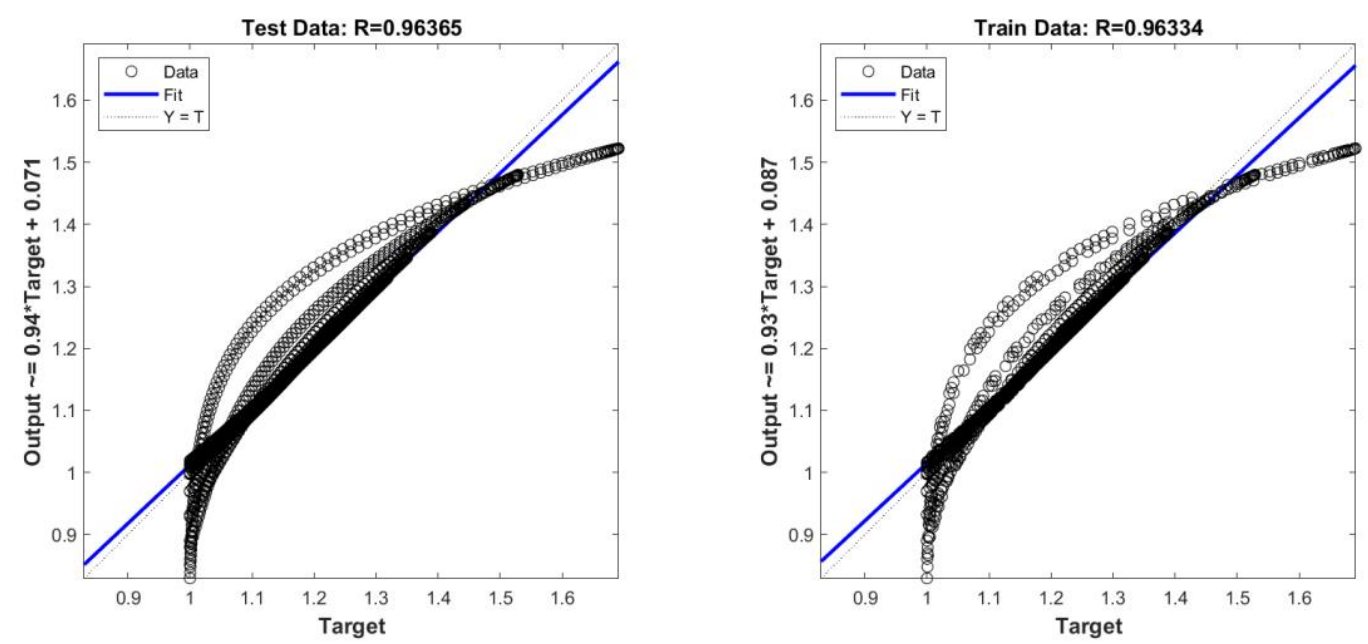
Figure 4. training and testing datasets for membership function gaussmf of input number 2

Figure 5 shows the point by point data comparison between ANFIS and finite difference method. As shown in the figure, in some points, ANFIS cannot capture the behavior. Nevertheless, in most of the points and when a low level of the dataset is reached, ANFIS has a good capability in prediction and can predict conditions similar to those by the finite difference method. After having compared the data, the level of training the data is changed to investigate how the AI algorithm is capable of achieving the intelligence.

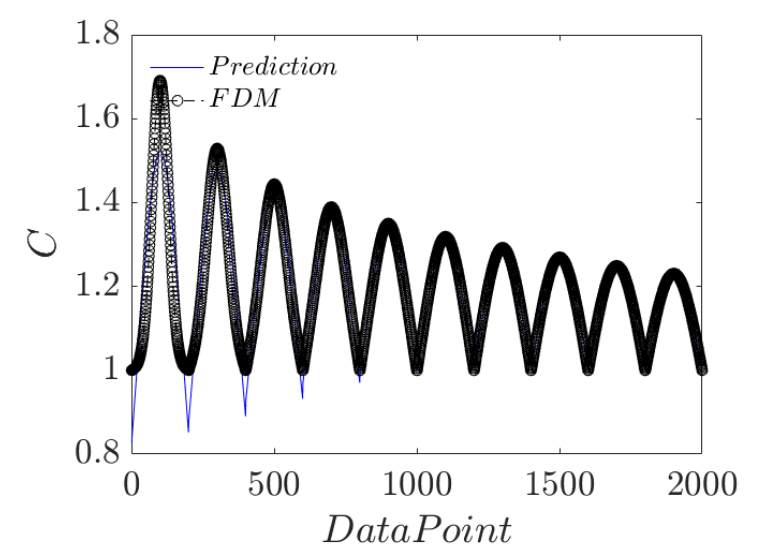

Figure 5. Datapoint comparison for epoch 10 membership functiotabn gaussmf of input number 2

Figure 6 shows that in the beginning, 1 percent of the data is used. As shown, the errors in both testing and training for MSE and RMSE criteria start to decrease in the beginning. By increasing the iterations, specifically in testing, the criteria increase, meaning that this method cannot reach intelligence by using one percent of the data. 

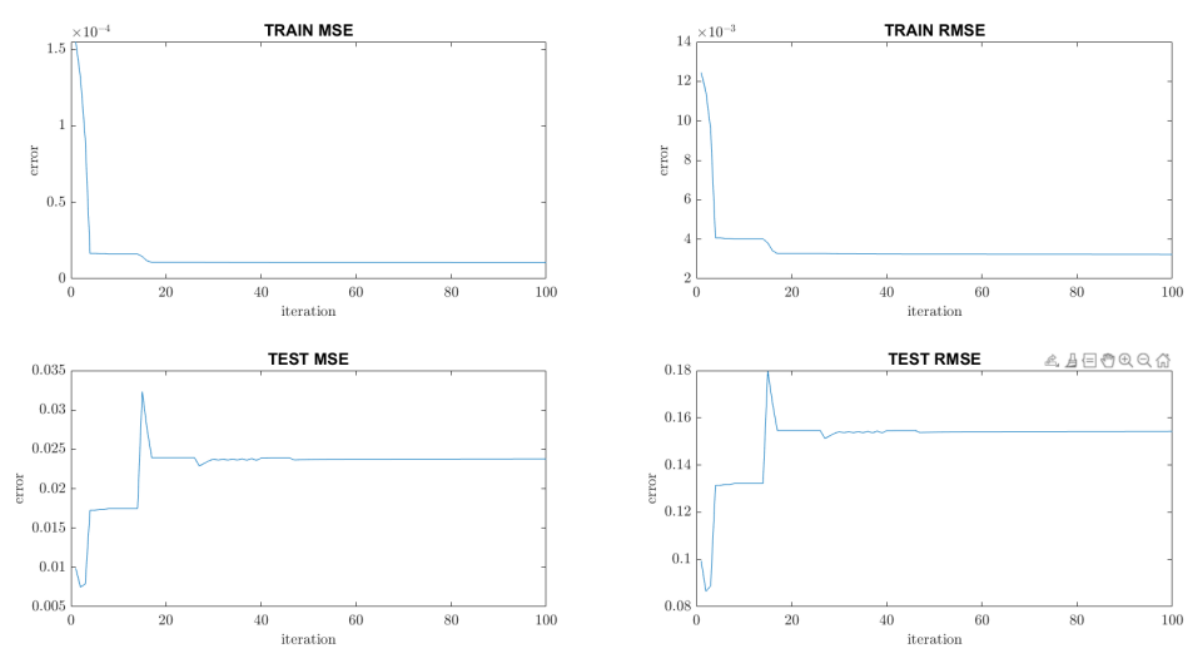

Figure 6. Error analysis of $1 \%$ of data sets for MSE and RMSE criteria in both training and testing

Figure 7 indicates that the percentage of the data in training increases to 5 percent. As shown in both testing and training stages, the errors decrease, and after 50 iterations, the errors converge in both training and testing regarding both SME and RMSE. It is also worth mentioning that each iteration denotes a simulation, meaning that 50 iterations refer to the fact that 50 models are studied.
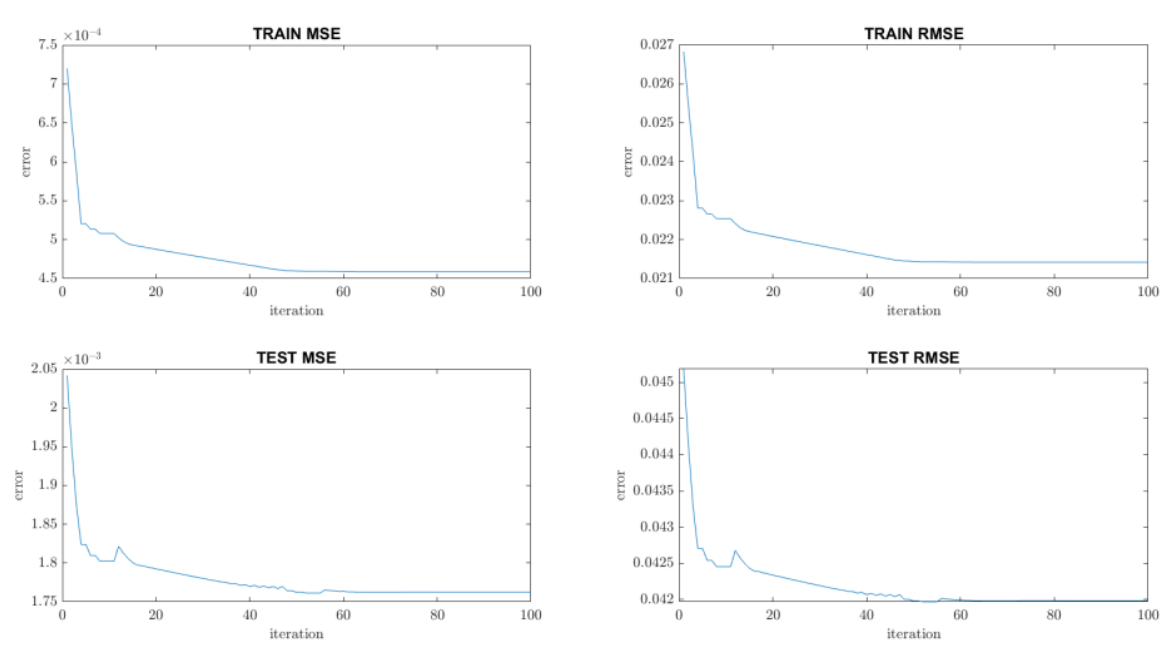

Figure 7. Error analysis of 5\% of data sets for MSE and RMSE criteria in both training and testing 
Figure 8 shows that the percentage of data increases to 10 percent, and as shown, the errors decrease. Furthermore, again, in the beginning, the errors decrease in the first epoch or iteration. Furthermore, after 40 to 45 iterations or epochs, the final convergence is reached, and the method and prediction can be relied on.
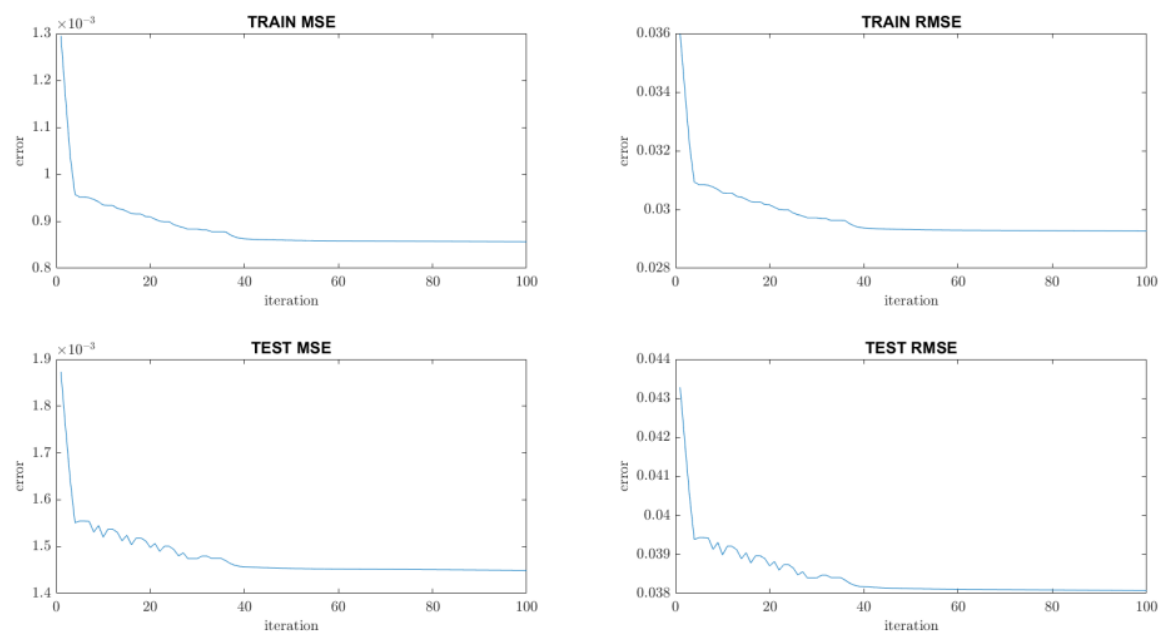

Figure 8. Error analysis of $10 \%$ of data sets for MSE and RMSE criteria in both training and testing

For a more accurate comparison, BNN technique is also used to train the prediction of diffusion as a function of time and domain. In terms of prediction capabilities, this approach is comparable to ANFIS method. In this instance, ANFIS method includes four membership functions with 200 iterations, denoting the technique's best performance. This method is compared with BNN method for the evaluation of the prediction capability. Figure 9 demonstrates that ANFIS technique predicts diffusion slightly better than BNN method. In this respect, ANFIS method returns R>0.99, while BNN approach yields $\mathrm{R}$ around 0.98 . For various time steps, both machine learning approaches are accurate enough to estimate diffusion throughout the domain. Figure 10 shows a comparison of FDM, ANFIS, and BNN methods. The results show that ANFIS method can accurately trace diffusion concentration throughout the domain. In addition, BNN 
technique does not respond well to the boundary condition, while ANFIS method can properly estimate the concentration near the boundary condition. By increasing the number of layers in $\mathrm{BNN}$, this technique can achieve higher numerical precision.
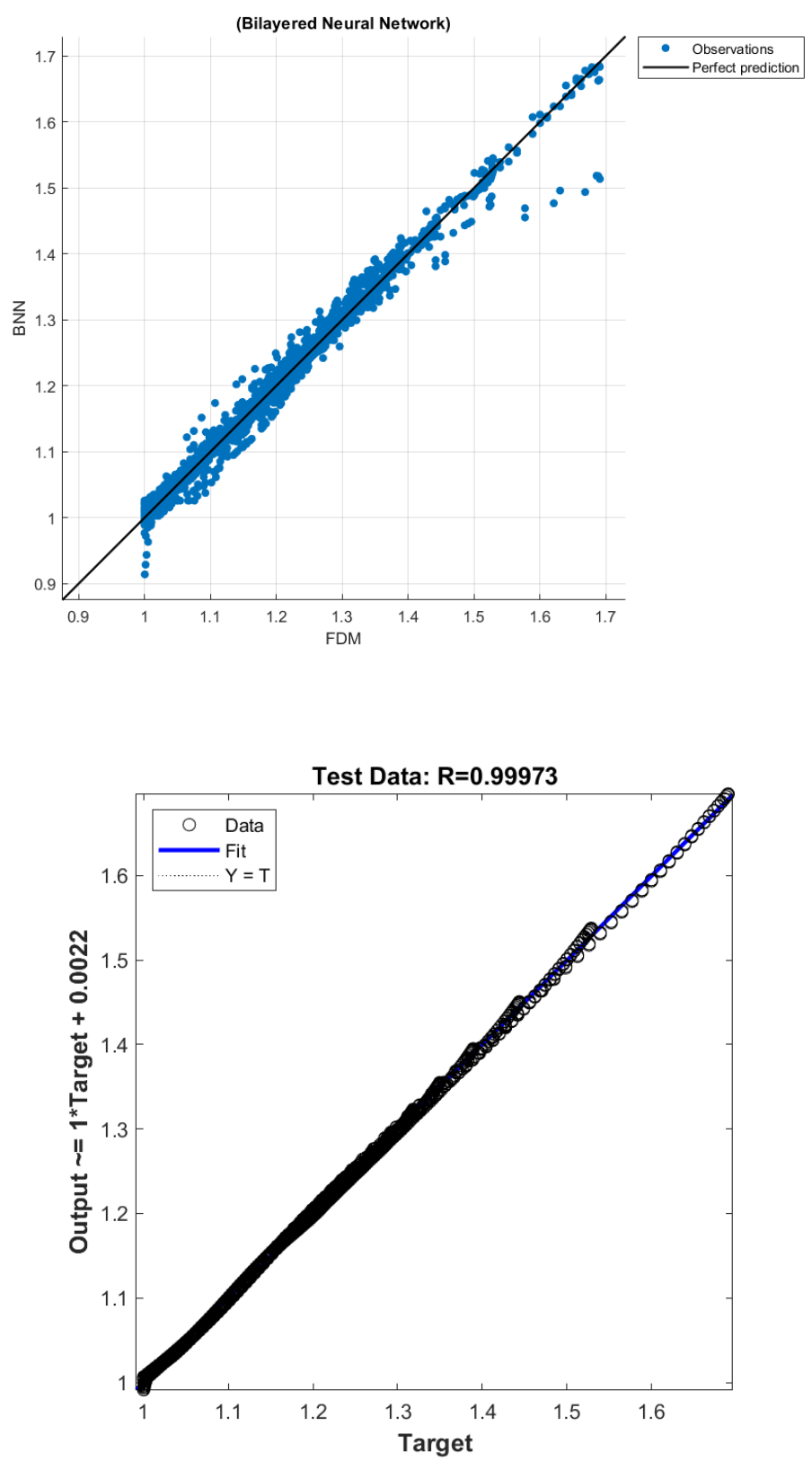

Figure 9. Comparison between BNN and ANFIS methods. 


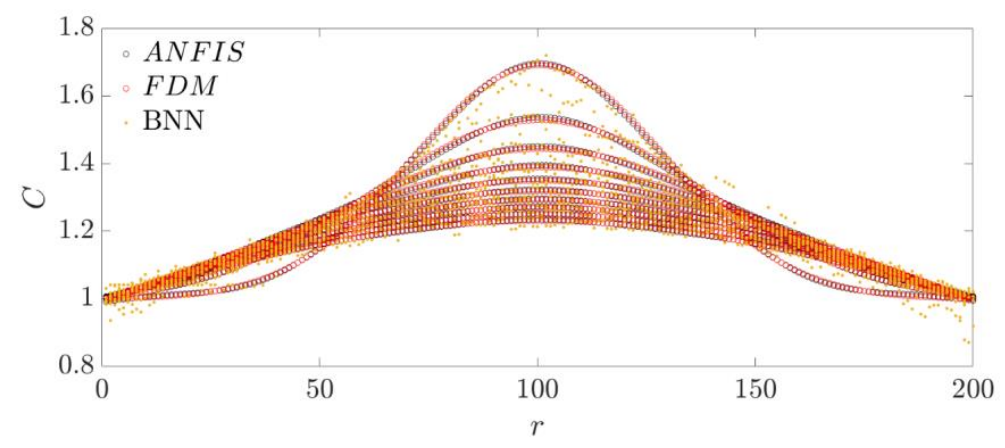

Figure 10. Comparison between BNN, ANFIS \& FDM across the domain for different time steps

In this research, all ANFIS method parameters are chosen based on sensitivity analysis to determine the effect of each tuning parameter on the model's accuracy. For example, the number of iterations is determined based on MSE and RMSE computations, and the best model is picked based on a low RMSE. Furthermore, various amounts of data are chosen to test the model's learning capacity, and it is found that with just $10 \%$ of total data sets, this model is capable of predicting the process. A smaller number of datasets can have a detrimental effect on numerical computation convergence. The findings also indicate that this process's numerical data sets can be learned in AI framework with a low rate of numerical instability.

\section{Conclusion}

In the current research, a combination of FDM and machine learning is used to predict the diffusion for low and high concentration flows across the domain for different time steps. This mathematical integration results in faster computation diffusion in the domain based on a machine learning algorithm. For the machine learning method, ANFIS model is used for datasets. Three time-dependent input parameters are considered, and the final 
concentration is used as the output of the model. The results show that the machine learning model can present similar results to FDM method with a lower computational time. There is a good agreement between FDM and machine learning for low and high concentration domains and boundary conditions. With $5 \%$ of datasets, ANFIS can fully predict the diffusion phenomena. With 50 iterations, ANFIS method can be fully trained to predict the diffusion for low and high concentrations. This numerical technology can be used to facilitate the diffusion of molecules in aqueous solutions in the domain of membrane structure and improve the efficiency of chemical engineering processes. To understand the membrane and diffusion process, more input parameters can be used for training of machine learning methods.

For a more accurate comparison, BNN method is also utilized for training the prediction of diffusion processes. This technique is similar to ANFIS method in terms of prediction capability. The findings show that ANFIS approach outperforms BNN method in predicting diffusion. In this regard, ANFIS technique gives $\mathrm{R}>0.99$ while BNN method yields $\mathrm{R}$ around 0.98 . Both machine learning methods are accurate enough to predict diffusion throughout the domain for different time steps. BNN approach does not perform well at the boundary condition while ANFIS method accurately estimates the concentration at the boundary condition.

Besides, it is worth mentioning that AI can find the relationship between inputs and the outputs and determine which input has the primary influence on the output of the study to optimize the process. In future studies, AI and other methods can be used for faster predictions and optimization processes. Furthermore, the study also faces some limitations. Since supervised machine learning methods are data-driven, the methods are trained based on the inputs and AI can predict other inputs which are in that particular 
range. Thus, in the prediction phase, only the condition that is similar can be predicted, and consequently, the relationship between inputs and outputs can be found via AI. AI can be used with other methods in different processes. This technology can be used whenever the researchers face time and cost limitations. As a conclusion, AI can be used as a commentary method besides the numerical methods to make the prediction faster.

\section{References}

Asadollahzadeh, M., Raoufi, N., Rezakazemi, M., \& Shirazian, S. (2018). Simulation of nonporous polymeric membranes using CFD for bioethanol purification. Macromolecular Theory and Simulations, 27(3), 1700084.

Babanezhad, M., Masoumian, A., Nakhjiri, A. T., Marjani, A., \& Shirazian, S. (2020). Influence of number of membership functions on prediction of membrane systems using adaptive network based fuzzy inference system (ANFIS). Scientific Reports, 10(1), 1-20.

Dai, Z., Noble, R. D., Gin, D. L., Zhang, X., \& Deng, L. (2016). Combination of ionic liquids with membrane technology: A new approach for $\mathrm{CO} 2$ separation. Journal of Membrane Science, 497, 1-20.

Marjani, A., Nakhjiri, A. T., Adimi, M., Jirandehi, H. F., \& Shirazian, S. (2020). Effect of graphene oxide on modifying polyethersulfone membrane performance and its application in wastewater treatment. Scientific Reports, 10(1), 1-11.

Najafi, B., Faizollahzadeh Ardabili, S., Shamshirband, S., Chau, K.-w., \& Rabczuk, T. (2018). Application of ANNs, ANFIS and RSM to estimating and optimizing the parameters that affect the yield and cost of biodiesel production. Engineering Applications of Computational Fluid Mechanics, 12(1), 611-624. https://doi.org/doi.org/10.1080/19942060.2018.1502688

Padaki, M., Murali, R. S., Abdullah, M. S., Misdan, N., Moslehyani, A., Kassim, M., Hilal, N., \& Ismail, A. (2015). Membrane technology enhancement in oil-water separation. A review. Desalination, 357, 197-207.

Pishnamazi, M., Babanezhad, M., Nakhjiri, A. T., Rezakazemi, M., Marjani, A., \& Shirazian, S. (2020). ANFIS grid partition framework with difference between two sigmoidal membership functions structure for validation of nanofluid flow. Scientific Reports, 10(1), 1-11.

Purkait, M. K., \& Singh, R. (2018). Membrane technology in separation science. CRC Press.

Razavi, R., Sabaghmoghadam, A., Bemani, A., Baghban, A., Chau, K.-w., \& Salwana, E. (2019). Application of ANFIS and LSSVM strategies for estimating thermal conductivity enhancement of metal and metal oxide based nanofluids. Engineering Applications of Computational Fluid Mechanics, 13(1), 560-578. 
Razavi, S. M. R., Shirazian, S., \& Nazemian, M. (2016). Numerical simulation of CO2 separation from gas mixtures in membrane modules: Effect of chemical absorbent. Arabian Journal of Chemistry, 9(1), 62-71.

Rezakazemi, M., Dashti, A., Asghari, M., \& Shirazian, S. (2017). H2-selective mixed matrix membranes modeling using ANFIS, PSO-ANFIS, GA-ANFIS. International Journal of Hydrogen Energy, 42(22), 15211-15225.

Rezakazemi, M., Mosavi, A., \& Shirazian, S. (2019). ANFIS pattern for molecular membranes separation optimization. Journal of Molecular Liquids, 274, 470-476.

Rezakazemi, M., Shirazian, S., \& Ashrafizadeh, S. N. (2012). Simulation of ammonia removal from industrial wastewater streams by means of a hollow-fiber membrane contactor. Desalination, 285, 383-392.

Sefeedpari, P., Rafiee, S., Akram, A., Chau, K., \& Komleh, S. (2015). Modeling energy use in dairy cattle farms by applying multi-layered adaptive neuro-fuzzy inference system (MLANFIS). International journal of dairy science.

Yan, Y., Safdari, A., \& Kim, K. C. (2020). Visualization of nanofluid flow field by adaptive-network-based fuzzy inference system (ANFIS) with cubic interpolation particle approach. Journal of Visualization, 1-9. 